\title{
Characterisation of the mechanical behaviour of a polyurethane elastomer based on indentation and tensile creep experiments
}

\author{
B. Buffel ${ }^{1}$, K. Vanstreels ${ }^{2}$, F. Desplentere ${ }^{1}$, B. Dekeyser ${ }^{3}$ \\ \& I. Verpoest ${ }^{4}$ \\ ${ }^{1}$ KHBO Expertisecentrum Kunststoffen, KU Leuven-KHBO, Belgium \\ ${ }^{2}$ imec, Belgium \\ ${ }^{3}$ International Development Centre, Recticel N.V., Belgium \\ ${ }^{4}$ Department of Metallurgy and Materials Engineering, \\ KULeuven, Belgium
}

\begin{abstract}
This research focuses on the determination of the mechanical properties of a viscoelastic polyurethane material with 2 different measuring techniques on 2 different length scales. Instrumented Indentation Testing (IIT) was used to test the material on a micro scale while tensile creep experiments characterised the macro scale material behaviour. All experimental data were processed by means of a fitting procedure based on the standard linear solid material model. The experiments were performed with different loading rates and hold values. The developed fitting procedure proved to be applicable to analyse the experimental data on both length scales. FEM was used to coordinate the applied strains of both measuring techniques. A comparison between the results originating from the experiments with both techniques indicated a stiffer material response on the micro scale (up to $4 \mathrm{x}$ ). The more complex strain field inside the material during indentation compared to the uniform tensile loading on macro scale is responsible for this large discrepancy. For this reason comparing the results of IIT with tensile creep results should be done with great care.

Keywords: instrumented indentation testing, creep experiments, standard linear solid material model, polyurethane elastomer.
\end{abstract}




\section{Introduction}

The worldwide interest in elastomeric materials has grown significantly during the last decades. Applications can be found in various areas such as automotive and construction. Together with the increasing importance of elastomers, a higher demand for quantitative material data arose. Despite the fact that the first non-linear material models date back to the $19^{\text {th }}$ century, the measurement of quantitative material parameters still remains a key issue regarding the modelling of viscoelastic materials.

Elastomeric materials can be produced in a large window of material properties. These properties originate from the chemical and physical interactions between the polymer chains which depend on the structure and composition of the chains. In this way not only the raw materials but also the recipe and preparation method influences the final properties of elastomeric materials. Because of this high number of influencing parameters High Throughput Experiments (HTE) are helpful to accelerate the investigations on different elastomeric formulation and preparation methods. HTE allows a high degree of automation of the production and testing of multiple elastomers which is essential for fast and sustainable research (Kranenburg et al. [1], Majoros et al. $[2])$.

Instrumented Indentation Testing (IIT) uses a probe with a spherical or pyramidal tip to indent a sample on a micro or nano scale. During the indentation a force-displacement curve is recorded which is processed afterwards to extract the material parameters (Fischer-Cripps [3]). IIT can be used to characterize the mechanical properties of a wide range of materials. This range includes polymers and elastomers with a distinct time dependent mechanical response. (Galli et al. [4], Vanlandingham et al. [5]). For this type of materials the standard elasticplastic analysis (Oliver and Pharr [6]) is insufficient and can be replaced by more powerful methods to characterize linear viscoelastic material behaviour (FischerCripps [7], Oyen [8, 9]). These methods were used within the scope of this research and adapted to tensile creep experiments on macro scale.

Conventional methods like bending or tensile testing are considered as the bottleneck in HTE. To overcome this problem IIT is often applied in a HTE workflow as it is capable of capturing experimental data in a fast and accurate way. In combination with the ability to test viscoelastic materials, IIT was selected to test the polyurethane elastomer within this research. This research focuses on the comparison of IIT to conventional tensile creep experiments in order to validate the measuring technique. The standard linear solid model was used to capture the non-linear material behaviour (Fischer-Cripps [7], Powel [10]).

\section{Experimental methods}

\subsection{Micro scale testing: instrumented indentation testing}

Indentation tests were performed at the imec Research Institute with a NanoIndenter XP (MTS Systems) on a polyurethane elastomer film produced by 
Recticel (IDC Wetteren Belgium). The film had an average thickness of 1,7mm. The indenter tip was a sphere with a radius $\mathrm{R}=250 \mu \mathrm{m}$. The indentation experiments consisted out of a force controlled loading phase with a loading rate of $0,5 \mathrm{mN} / \mathrm{s}$ up to a varying peak force $\left(\mathrm{F}_{\max }=0,5-1-2-5-10-20 \mathrm{mN}\right)$. In another set of experiments the peak force was fixed $\left(\mathrm{F}_{\max }=2 \mathrm{mN}\right)$ with varying loading rates $(\mathrm{LR}=0,05-0,3-0,5-0,75-1 \mathrm{mN} / \mathrm{s})$.

The governing equation for spherical indentation in an elastic medium is (Johnson [11]):

$$
h^{3 / 2}=\frac{3}{4 \sqrt{R}} F \frac{1-v}{E}
$$

where $\mathrm{h}$ and $\mathrm{F}$ are indentation depth and load respectively. During a force controlled loading phase of an indentation test $(\mathrm{F}(\mathrm{t})=\mathrm{k} . \mathrm{t})$ and in the case of an incompressible solid $(v=0,5)$ this equation can be written as:

$$
h^{3 / 2}(t)=\frac{3 k}{8 \sqrt{R}} \frac{t}{2 G}
$$

in which $\mathrm{G}$ is the shear modulus of the material. Due to the non-linear material behaviour the experimental time-displacement data were processed based on the procedure developed by M. Oyen (Oyen $[8,9]$ ) in which the standard linear solid model describes the non-linear material behaviour by means of a parallel springdashpot system in series with a second spring. The procedure resulted in the fitting of the loading curve with the following equation:

$$
\begin{gathered}
h^{3 / 2}(t)=B_{0} t-B_{1}\left(1-e^{-t / \tau_{1}}\right) \\
\text { with } B_{0}=\frac{3 k}{8 \sqrt{R}} C_{0} \quad \text { and } \quad B_{1}=\frac{3 k}{8 \sqrt{R}} C_{1} \tau_{1}
\end{gathered}
$$

The $\mathrm{C}_{0}$ end $\mathrm{C}_{1}$ values obtained from the fitting procedure are used to calculate the shear and Young's modulus of the polyurethane elastomer:

$$
\begin{gathered}
G_{0}=\frac{1}{2\left(C_{0}-C_{1}\right)} \text { and } G_{\infty}=\frac{1}{2 C_{0}} \\
E_{0}=2 G_{0}(1+v) \text { and } E_{\infty}=2 G_{\infty}(1+v)
\end{gathered}
$$

These values are limiting values for the immediate response $\left(G_{0}\right.$ and $\left.E_{0}\right)$ and the response with the lapse of time $\left(\mathrm{G}_{\infty}\right.$ and $\left.\mathrm{E}_{\infty}\right)$. The ratio of both is a measure for the viscoelasticity of the material. The more this ratio approaches one, the more elastic the material behaves.

\subsection{FE-simulations}

In order to coordinate the experimental conditions of the micro scale testing on the macro scale tensile testing a FE model of the indentation experiment was build. Siemens NX 8.0 software coupled to a NASTRAN solver was used to build and solve the FE model. Due to symmetry, the model was limited to a quarter of the specimen and a part of the indenter sphere (fig. 1). The main goal of the model was to estimate the occurring strains in the indentation test in order to apply analogue strains in the tensile creep experiments on macro scale. 

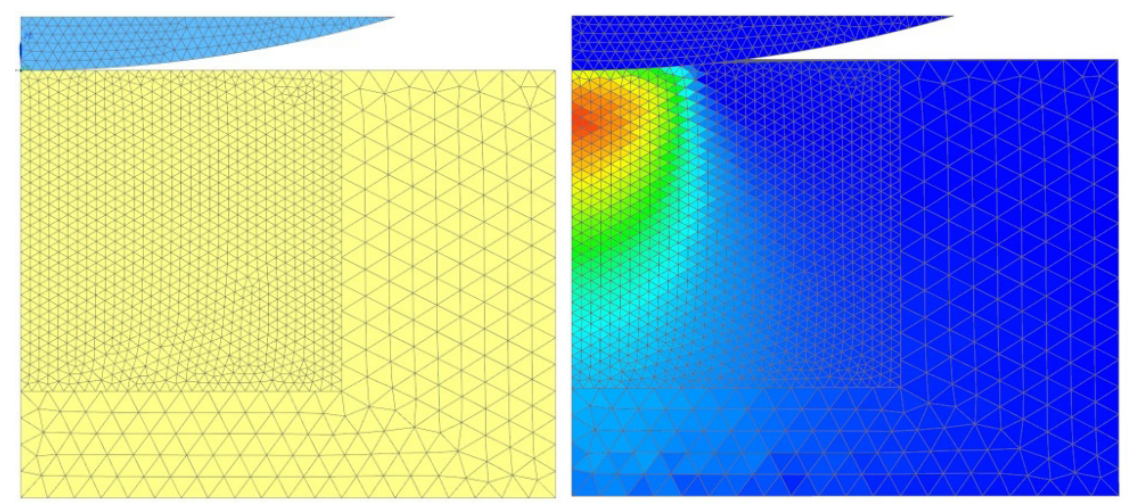

Figure 1: FE indentation model with refined mesh in the high strain zone (left). Strains in the vertical direction inside the specimen reaching a maximum and fading out deeper in the material (right).

Multiple simulations with varying indentation depths were performed with different coefficients of friction between the indenter tip and the sample surface. The highest strains were observed in the vertical direction of indentation. These strains imply the highest loading conditions on the material and were transferred to the macro tensile test. The results of these simulations are presented in figure 2. No significant influence of the coefficient of friction was observed.

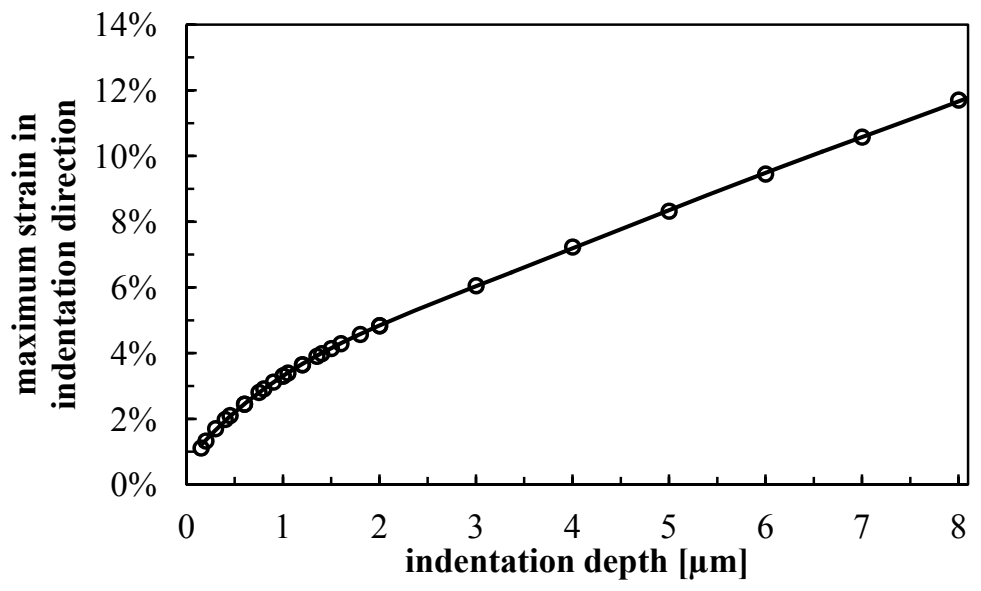

Figure 2: Maximum occurring strain in the indentation direction as a function of the indentation depth. Radius of the indenter sphere $=250 \mu \mathrm{m}$.

\subsection{Macro scale testing: tensile creep experiments}

The tensile experiments were performed on an Instron 3345 single column testing system equipped with a $5 \mathrm{kN}$ load cell and an initial clamping distance of 
$55 \mathrm{~mm}$. Dog bone shaped tensile test specimens were punched out of the same film as the indentation test sample. Experiments were performed at a fixed loading rate of $10 \mathrm{~N} / \mathrm{min}$ up to a varying hold value of $0,33-0,88-1,1-2,64-$ $3,63 \mathrm{~mm}$. These displacements can be linked to the occurring strains in the indentation direction via the FE-simulations, fig. 2. In a second series of tensile tests the peak force was fixed at $=50 \mathrm{~N})$ and the loading rate was varied $(\mathrm{LR}=5-$ $10-50-100-500 \mathrm{~N} / \mathrm{min}$ ). In both series the loading phase was followed by a 300s long holding phase in which the force was kept constant at the peak force $\mathrm{F}_{\text {max }}$.

The experimental macro scale tensile creep data were fitted to the same viscoelastic material model as the indentation test data. Therefore, the fitting procedure presented in equations (3)-(5) was adapted for the loading phase of the tensile test:

$$
\Delta l(t)=\frac{l_{0} k}{A(1+v)}\left[C_{0}-C_{1} \tau_{1}\left(1-e^{-t / \tau_{1}}\right)\right]
$$

In which $1_{0}, \Delta \mathrm{l}$ and $\mathrm{A}$ are respectively the initial clamping distance, displacement and cross sectional area of the specimen. During the constant force holding phase the material model is adapted to:

$$
\Delta l(t)=\frac{l_{0} k}{A(1+v)}\left[C_{0} t_{\text {rise }}-C_{1} \tau_{1} e^{-t / \tau_{1}}\left(e^{t_{\text {rise }} / \tau_{1}}-1\right)\right]
$$

with $t_{\text {rise }}=$ time to reach the hold value. Equations (6)-(8) can again be used to calculate the material stiffness values based on the outcome of the fitting procedure.

\subsection{Temperature dependency}

Due to different laboratory conditions the dependency of the mechanical properties on temperature was taken into account in order to make a comparison between macro and micro testing results. The glass transition temperature region was determined with a Q200 MDSC (TA Instruments) apparatus. A constant temperature increase of $10^{\circ} \mathrm{C} / \mathrm{min}$ from $-50^{\circ} \mathrm{C}$ up to $80^{\circ} \mathrm{C}$ was used. The results indicated a broad glass transition region between $5^{\circ} \mathrm{C}$ and $35^{\circ} \mathrm{C}$.

The dependency of material stiffness on temperature was measured using a tensile set up inside a Q800 DMA (TA Instruments). 3 samples were tested with a clamping distance and width of respectively 17,6 and 2,6mm. A frequency of $1 \mathrm{~Hz}$ and an amplitude of $15 \mu \mathrm{m}$ were applied. The results of the DMA temperature sweep are presented in figure 3 .

The stiffness of the polyurethane film possesses a very high dependency on temperature in the region around room temperature. Since the indentation laboratory is conditioned at $23^{\circ} \mathrm{C}$ this was taken as the reference situation and all experimental tensile creep results will be corrected with respect to this condition. 




Figure 3: Normalised stiffness of the polyurethane film as a function of temperature. The stiffness result at $23^{\circ} \mathrm{C}$ was taken as a reference.

\section{Results and discussion}

\subsection{Micro scale testing: instrumented indentation testing}

In contrast to the macro scale testing, no holding phase results could be obtained during the IIT due to practical limitations of the measuring device. Therefore $\mathrm{E}_{0}$ and $\mathrm{E}_{\infty}$ were extracted from the loading phase of the measurement. The results are presented as a function of hold value and loading rate in figures 4 and 5 respectively. $E_{0}$ is independent of the hold value up to a strain of $4 \%$. At larger strains the value of $\mathrm{E}_{0}$ decreases because the mathematical curve fitting optimisation is influenced by the data and larger strains which do not anymore correlate to the initial stiffness $E_{0}$. The decreasing trend in $E_{\infty}$ as a function of the hold value can be explained by the increasing viscous-plastic material behaviour as the indenter moves into the sample.

Figure 5 shows an increase of $\mathrm{E}_{0}$ and $\mathrm{E}_{\infty}$ with increasing loading rate. This observation, a stiffer material response with increasing loading rate, is often induced by the higher resistance to make polymer chains move across each other at higher rates. Comparing the values of $\mathrm{E}_{0}$ versus $\mathrm{E}_{\infty}$ clearly indicates the need for a non-linear material model. This proves that the selected loading rates were not too fast with respect to the material time constants. Due to the dependency of the fitted parameters on experimental conditions, future work is required to optimise the measuring methods and obtain absolute material properties. The large standard deviations presented by the error bars in the graphs will be discussed in section 3.3. 


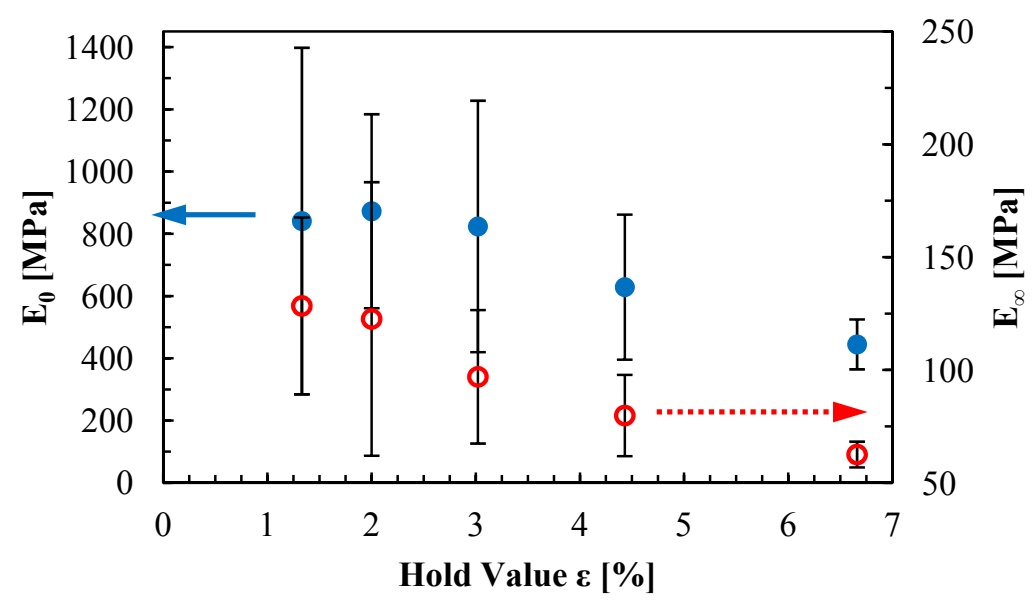

Figure 4: $\quad \mathrm{E}_{0}$ and $\mathrm{E}_{\infty}$ values as a function of the hold value applied during the indentation experiments. This value is expressed as the corresponding strain which was calculated via FEM. Filled circles represent $\mathrm{E}_{0}$; Empty circles represent $\mathrm{E}_{\infty}$.



Figure 5: $\quad \mathrm{E}_{0}$ and $\mathrm{E}_{\infty}$ values as a function of the loading rate of the indentation experiments. Filled circles represent $\mathrm{E}_{0}$ while empty circles represent $\mathrm{E}_{\infty}$.

\subsection{Macro scale testing: tensile creep experiments}

Figures 6 and 7 present $\mathrm{E}_{0}$ and $\mathrm{E}_{\infty}$ as a function of the hold value. In both figures data obtained from the loading phase (eqn. (9)) are distinguished from the data obtained from the holding phase (eqn. (10)). All data were corrected for the laboratory conditions based on the temperature dependency presented in fig. 3 . The error bars in the figures indicate the standard deviations out of 5 independent 
measurements. In almost all cases the results from the holding phase are more accurate. Moreover, the total sum of the residual values of the fitted curve vs. the experimental curve proved to be much lower in the case the holding phase of the curve was used to determine the stiffness values.

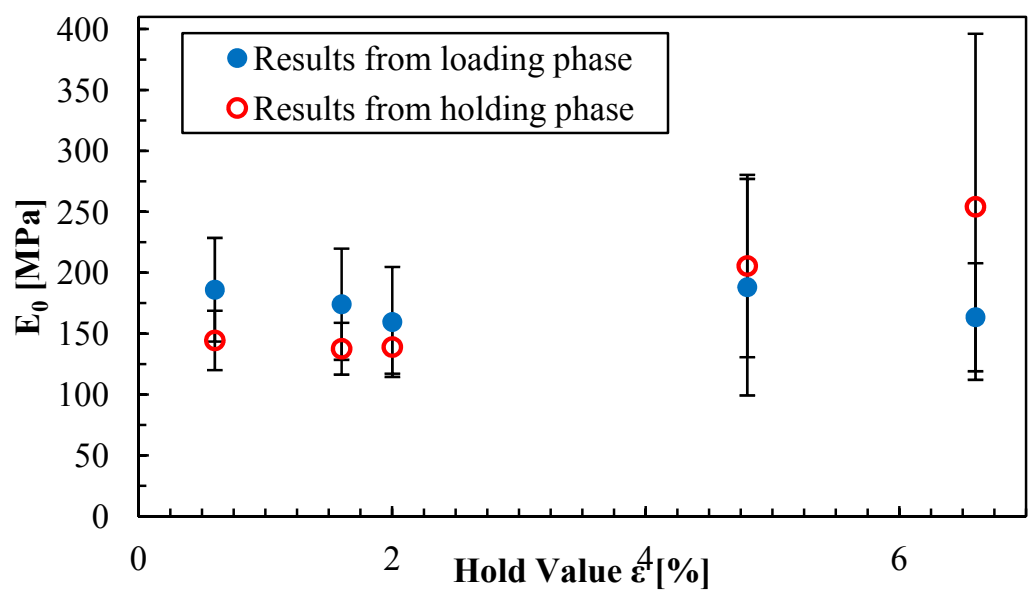

Figure 6: $\quad E_{0}$ values as a function of the hold value based on experimental tensile test data originating from the loading or holding phase. All experiments were performed at $10 \mathrm{~N} / \mathrm{min}$. The hold value is expressed as the corresponding strain (fig. 2) which was calculated via FEM.



Figure 7: $\quad E_{\infty}$ values as a function of the hold value based on experimental tensile test data originating from the loading or holding phase. All experiments were performed at $10 \mathrm{~N} / \mathrm{min}$. The hold value is expressed as the corresponding strain (fig. 2) which was calculated via FEM. 
In correspondence to the IIT results, there is no significant dependency of $\mathrm{E}_{0}$ on the hold value. Due to an increasing viscous-plastic behaviour of the elastomeric material, the values of $\mathrm{E}_{\infty}$ decrease with increasing hold value. The ratio of $E_{\infty}$ on $E_{0}$ yields values in the range of 0,075 , which indicates the need for a non-linear material model.

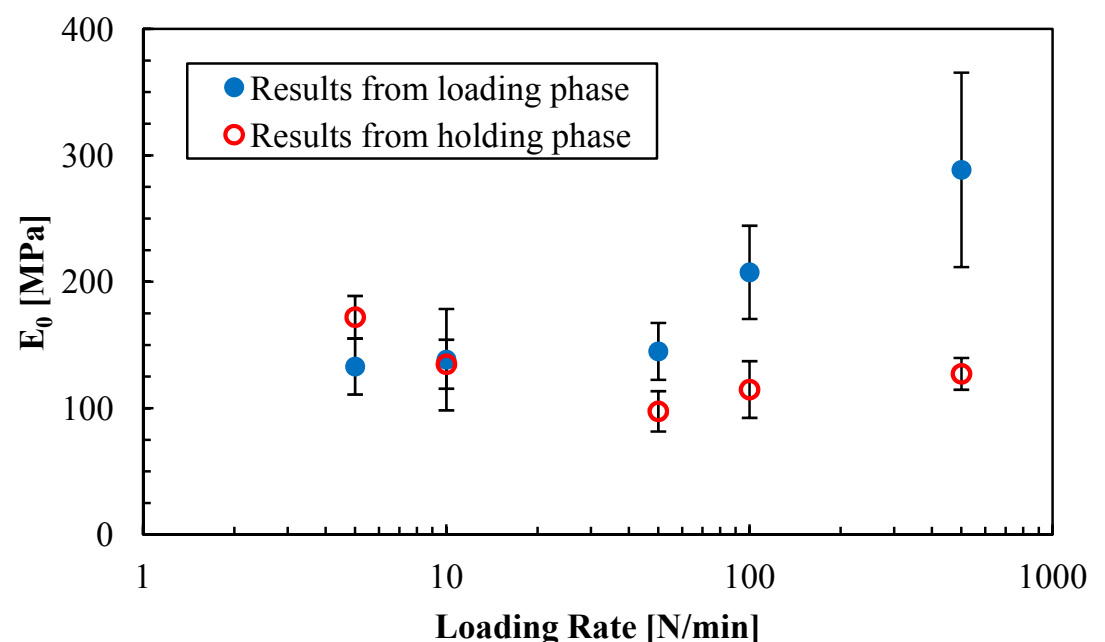

Figure 8: $\quad \mathrm{E}_{0}$ values as a function of the loading rate based on experimental tensile test data originating from the loading or holding phase. All tests were performed up to a hold value of $50 \mathrm{~N}$.



Figure 9: $\quad E_{\infty}$ values as a function of the loading rate based on experimental tensile test data originating from the loading or holding phase. All tests were performed up to a hold value of $50 \mathrm{~N}$. 
The dependency of $\mathrm{E}_{0}$ and $\mathrm{E}_{\infty}$ on a wide range of loading rates is shown in figures 8 and 9 . The results based on the loading phase of the curve show an increase of $E_{0}$ and $E_{\infty}$ with increasing loading rate. These results are again less accurate compared to the results from the holding phase as can be seen on the size of the error bars.

When $\mathrm{E}_{0}$ and $\mathrm{E}_{\infty}$ are extracted from the loading phase of the curve an increase in stiffness with increasing loading rate is observed. The increased resistance of polymer flow at higher rates explains this behaviour. This tendency is however not observed when data are extracted from the holding phase of the curve. This suggests that the stiffness values obtained from the holding part the curve are not sensitive to the preceding loading rate. The values for $E_{\infty} / E_{0}$ are situated in the range of 0,1 , which again indicates a distinct non-linear material behaviour.

\subsection{Comparison between both length scales}

When comparing the trends of $\mathrm{E}_{0}$ an $\mathrm{E}_{\infty}$ as a function of the hold value during the indentation experiments to the results of the macro tensile creep experiments a quite similar trend can be seen. When only the results from the loading phase are taken into account, the dependency of $\mathrm{E}_{0}$ an $\mathrm{E}_{\infty}$ on the loading rate is also quite comparable.

Considering the absolute stiffness values, the values obtained on the micro scale with IIT are significantly larger than the values obtained with tensile creep experiments on the macro scale. Figure 10 is an illustrative graph in which the average $E_{0}$ values determined on both length scales are compared to each other as a function of the hold value. The micro scale results are up to 4 times as stiff as the macro scale results. This stiffer response originates from the much more complex strain-fields during indentation testing compared to tensile testing (Johnson [7]). While the former method combines shear, tension and compression in one single indentation, tensile testing applies a single uniform loading condition. Comparing results of both experimental techniques should thus be done with great care.

The results obtained from the measurements on the macro scale are also much less dependent on experimental conditions than the results from IIT. The macro scale tests have the advantage of testing a larger volume of the sample material in one test and thus possess a higher averaging potential. This is in contrast to the IIT which tests a very small volume at the surface of the sample. This difference in test set up leads to a higher dependency of the fitted parameters on the testing conditions.

The standard deviations presented by the error bars in figures 4 and 5 are remarkably large. This high uncertainty can be explained by the sensitivity of the stiffness values on the fitted parameters. As can be seen in figure 11, a small change in $\mathrm{C}_{0}-\mathrm{C}_{1}$ has a large impact on $\mathrm{E}_{0}$ in the IIT-results region. Together with the inevitable mathematical error in the curve fitting algorithm this results in a large spread of the experimental data. Due to the different loading conditions and subsequent lower stiffness values, $E_{0}$ is less sensitive for the variation in $C_{0}-C_{1}$ in the tensile creep experiments. 




Figure 10: Comparison of $\mathrm{E}_{0}$ based on the holding phase of the tensile creep experiment on the macro scale (filled circle) and $\mathrm{E}_{0}$ determined with IIT on the micro scale (hollow circle).

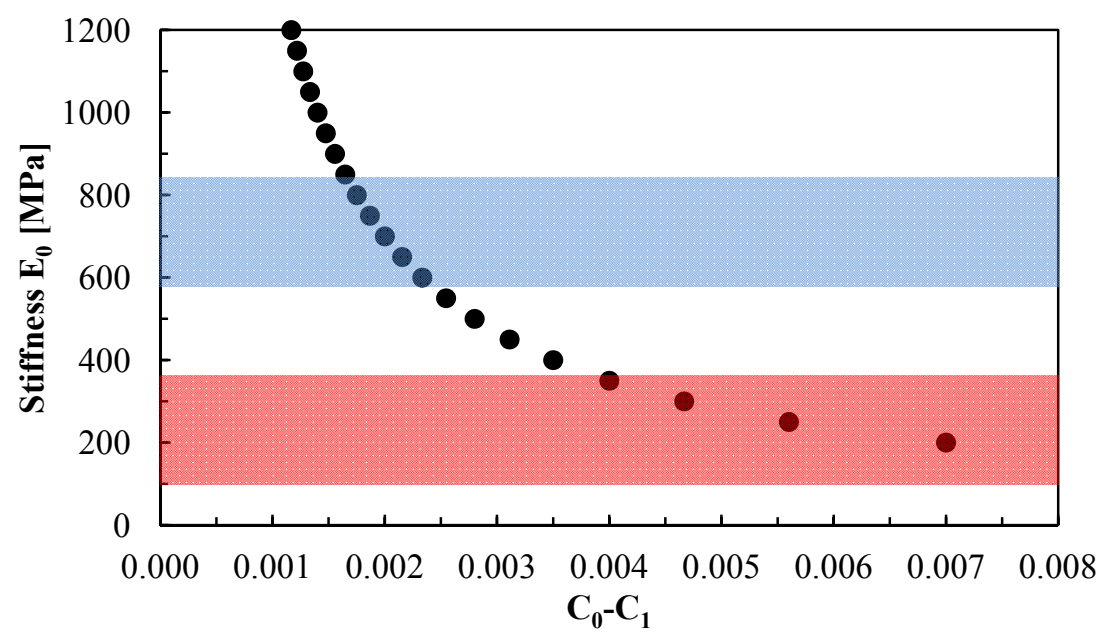

Figure 11: Sensitivity of $E_{0}$ on the difference between $C_{0}$ and $C_{1}$. The blue and red regions indicate the stiffness ranges in which the results of respectively the indentation and tensile experiments are situated. 


\section{Conclusions}

This research focuses on the characterisation of the stiffness properties of a polyurethane elastomer with 2 different experimental methods on 2 different length scales. As there is no single-valued elastic modulus for any elastomer, the limiting values of the elastic modulus $\mathrm{E}_{0}$ and $\mathrm{E}_{\infty}$ were determined within this research. In order to find these values, a curve fitting algorithm was used to fit experimental data to the standard linear solid material model. This was done on micro scale by making use of IIT and with macro scale tensile creep experiments.

FEM proved to be useful to coordinate the loading conditions on both length scales. Nonetheless the loading conditions of an indentation test can-not be transferred unambiguously to a tensile experiment. In an indentation test; the nature of the loading is a complex mixture of hydrostatic compression, tension and shear (Johnson [7]) while a tensile test applies a uniform tensional strain. This difference in loading condition induces a difference of a factor up to 4 between the results on both length scales.

In order to exclude the loading condition dependency, future research should focus on the optimization of the IIT. Useful steps would be an increase in data sampling rate or hold value and lower loading rates. In correspondence to the good results on macro scale, a creep measurement at constant load during an IIT is advisable.

\section{References}

[1] Kranenburg, J.M., van Duin, M., and Schubert S., Screening of EPDM cure states using depth-sensing indentation, Macromolecular Chemistry and Physics, 208, pp. 915-923, 2007.

[2] Majoros, L.I., Dekeyser, B., Haucourt, N., Castelein, P., Paul, J., Kranenburg, J.M., Rettler, E., Hoogenboom and R., Schubert, S., Preparation of polyurethane elastomers (PUEs) in a high-throughput workflow, Journal of Polymer Science: Part A: Polymer Chemistry, 49, pp. 301-313, 2011.

[3] Fischer-Cripps, A.C., Nanoindentation, Springer: NY, pp. 21-38, 2002.

[4] Galli, M., Comley, K.S.C., Shean, T.A.V. and Oyen, M.L., Viscoelastic and poroelastic mechanical characterization of hydrated gels. Journal of Materials Research, 24(3), pp.973-979, 2008.

[5] Vanlandingham, M.R., Chang N.-K., Drzal, P.L., White, C.C. and Chang, S.-H., Viscoelastic characterization of polymers using instrumented indentation. I. quasi-static testing. Journal of Polymer Science: Part B: Polymer Physics, 43, pp. 1794-1811, 2005.

[6] Oliver, W.C. and Pharr, G.M., An improved technique for determining hardness and elastic modulus using load and displacement sensing indentation experiments, Journal of Materials Research, 7(6), pp. 1564$1583,1992$. 
[7] Fischer-Cripps, A.C., A simple phenomenological approach to nanoindentation creep, Material Science and Engineering, 385, pp. 74-82, 2004.

[8] Oyen, M.L., Spherical indentation creep following ramp loading, Journal of Materials Research, 20(8), pp. 2094-2100, 2005.

[9] Oyen, M.L., Sensitivity of polymer nanoindentation creep measurements to experimental variables, Acta Materialia, 55, pp. 3633-3639, 2007.

[10] Powel, P.C., Engineering with polymers, Chapman and Hall London, 1983.

[11] Johnson, K.L., Contact Mechanics, Cambridge University Press, 1985. 